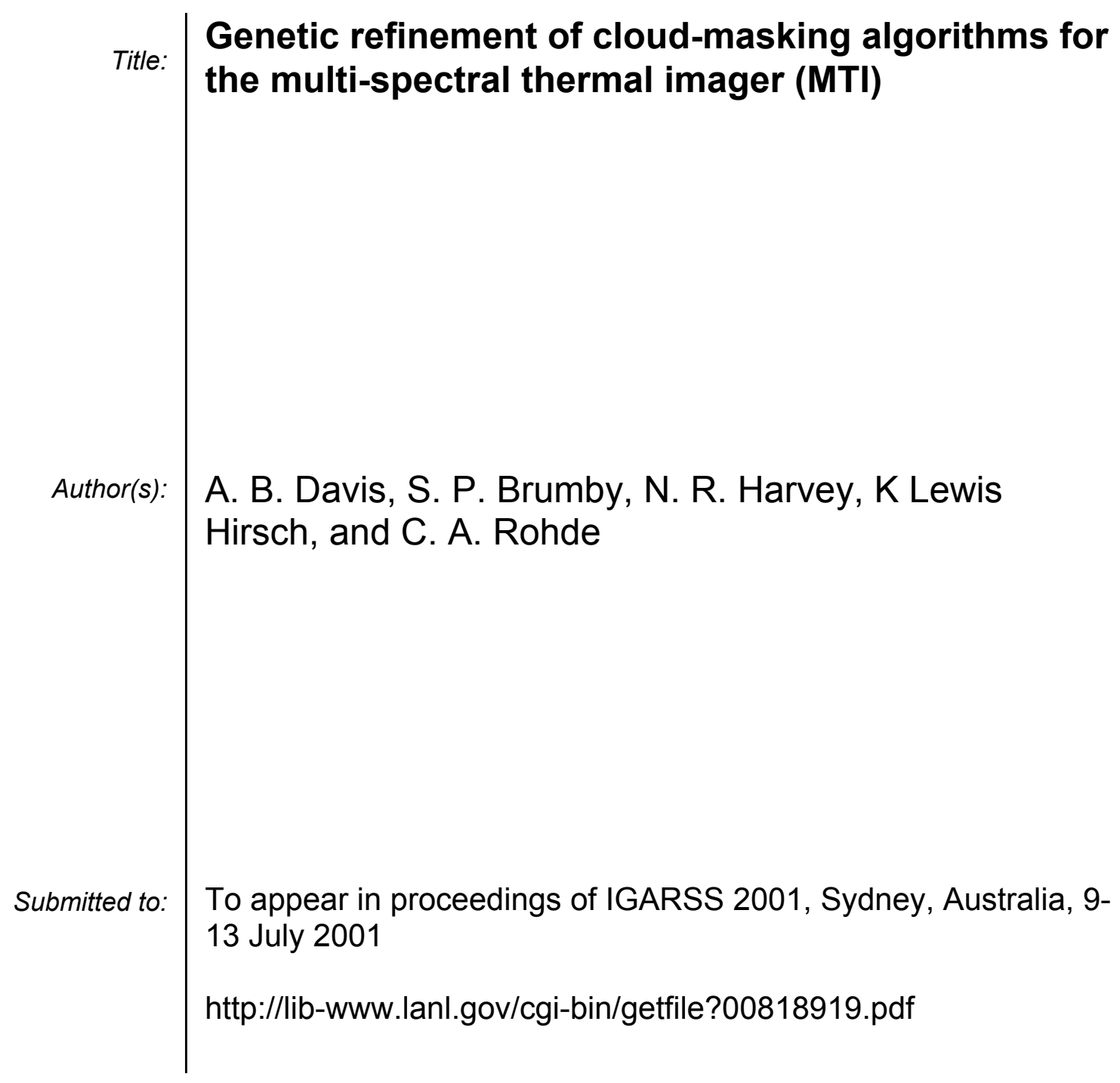




\title{
Genetic refinement of cloud-masking algorithms for the multi-spectral thermal imager (MTI)
}

\author{
A. B. Davis, S. P. Brumby, N. R. Harvey, K Lewis Hirsch, and C. A. Rohde \\ Space and Remote Sensing Science Group, Los Alamos National Laboratory, \\ Mail Stop D436, Los Alamos, New Mexico 87544, U.S.A.
}

\begin{abstract}
The Multi-spectral Thermal Imager (MTI) is a highperformance remote-sensing satellite designed, owned and operated by the U.S. Department of Energy, with a dual mission in environmental studies and in nonproliferation. It has enhanced spatial and radiometric resolutions and state-of-theart calibration capabilities. This instrumental development puts a new burden on retrieval algorithm developers to pass this accuracy on to the inferred geophysical parameters. In particular, the atmospheric correction scheme assumes the intervening atmosphere will be modeled as a plane-parallel horizontally-homogeneous medium. A single dense-enough cloud in view of the ground target can easily offset reality from the calculations, hence the need for a reliable cloud-masking algorithm. Pixel-scale cloud detection relies on the simple facts that clouds are generally whiter, brighter, and colder than the ground below; spatially, dense clouds are generally large on some scale. This is a good basis for searching multispectral datacubes for cloud signatures. However, the resulting cloud mask can be very sensitive to the choice of thresholds in whiteness, brightness, temperature, and connectivity. We have used a genetic algorithm trained on (MODIS Airborne Simulator-based) simulated MTI data to design a cloud-mask. Its performance is compared quantitatively to hand-drawn training data and to the EOS/Terra MODIS cloud mask.
\end{abstract}

\section{INTRODUCTION}

To take full advantage of the advanced spatial and radiometric resolutions and calibration capabilities of the Multi-spectral Thermal Imager (MTI) spacecraft [1], atmospheric effects such as water vapor absorption and aerosol scattering must be estimated and removed, and clouds, which limit seeing of the ground, must be identified and masked out from the images. Efforts are underway to develop algorithms to fully automate this processing. Qualitative results on have been reported in [2].

The MODIS Airborne Simulator (MAS) [3] is a high resolution scanning spectrometer mounted on an aircraft. Its primary objective is to support the MODerate resolution Imaging Spectroradiometer (MODIS) instrument on the EOS/Terra spacecraft. In the present work, spectrally resampled MAS data from the Smoke, Clouds, And Radiation - Brazil (SCAR-B) campaign is used as a proxy for MTI spacecraft data. We are interested in comparing several techniques for automatically masking out clouds, and will present results for a genetic algorithm approach (using a software package called GENIE) which attempts to evolve a cloud mask achieving automation, robustness, and good classification performance.

\section{METHOD COMPARISON}

Observationally, clouds are usually much brighter than their background, caused by refraction by the ice crystals and water droplets that make up the cloud. Clouds usually appear white when viewed from above. Due to the altitude of the clouds, they are cold, and when viewed from above they often appear much colder than their background. The MTI cloud mask algorithm uses these three basic to determine a pixel-scale cloud mask. MTI has 15 multi-spectral imagery (MSI) bands, including three visible bands and one near infrared (NIR) band each with a spatial resolution of $5 \mathrm{~m}$, and four NIR, two short-wave infrared (SWIR), two mid-wave infrared (MWIR) and three long-wave thermal IR bands each with a resolution of $20 \mathrm{~m}$ [4]. The MTI cloud mask algorithm uses bands "C" $(0.65 \mu \mathrm{m}$ visible red $)$, "E" $(0.88 \mu \mathrm{m}$ near IR),

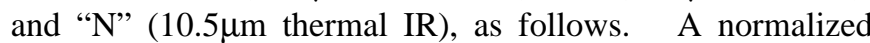
difference vegetation index (NDVI) of " $C$ " and " $E$ " is used to determine whiteness; the " $\mathrm{C}$ " band alone determines brightness; and the thermal " $\mathrm{N}$ " band is used to determine a relative temperature, using simple-thresholds on each of these. While these codes are mostly automated, the routines require that the thresholds be determined by hand, with a human user selecting representative cloudy pixels and thresholds in a false-color RGB display. These routine are currently part of MTI's Level 2 data processing for regular data retrievals.

Ackerman et al., [5] describe a physics-based cloud mask algorithm developed for the MODIS instrument using MAS data. Their automated routine masks pixels that contain optically thick aerosol, clouds, or shadow. It returns a 48 bit cloud mask which includes a two bit classification for the likelihood that a given pixel is cloudy. This algorithm uses radiance values in seventeen MAS bands, plus extensive ancillary data including instrument viewing geometry, a land/water/ice map, and an ecosystem map (land-cover classification for each pixel). While automated and quite robust, this algorithm is computationally expensive to run, and so we are interested in exploring a machine learning approach to attempt to evolve a simpler, but still automated and robust, alternative algorithm. 
GENIE [6] is an evolutionary computation software system that rapidly evolves automatic feature extraction (AFE) tools for multi-spectral imagery (MSI). GENIE is designed for a range of imagery analysis tasks, and is built to process and combine data from a wide variety of imagery sources (MSI, hyper-spectral imagery (HSI), and electro-optical (EO) imagery; using visible through thermal IR wavelengths).

GENIE, described at length elsewhere [7], uses a genetic algorithm to assemble image-processing tools (retrieval algorithms) from a collection of spatial, spectral, and spatiospectral image processing operators, including edge detectors, texture measures, band math operations, thresholds, and morphological filters. A population of candidate tools is generated, ranked according to a fitness metric measuring their performance on some user-provided training data, and fit members of the population permitted to reproduce. Each candidate tool in the population generates a number of intermediate feature planes, which are then combined using a supervised classifier backend (currently a Fisher discriminant and optimal threshold which minimizes the classification error) to generate a binary result mask. This process cycles until the population converges to a solution, or the user decides to accept the current best solution, or decides to change the training data.

\section{EVOLVING A SPATIO-SPECTRAL CLOUD MASK}

The MODIS cloud mask was run on part of a scene from the MAS SCAR-B flight series (95-163, track 7). Training data for GENIE was provided by manual mark-up of the scene. This is a straight-forward procedure for dense, optically-thick clouds, and not every pixel needs to be marked. More careful mark-up techniques would be needed to provide accurate training data for optically-thin clouds. Test data was produced by two techniques: manual classification of a complete MAS flight track, and the output of the MODIS cloud-mask, which is taken to be a "gold standard" for this problem.

A population of 50 candidate tools, each consisting of 20 primitive image processing steps, was evolved for 30 generations. This evolution required approximately 11 minutes of wall-clock time running on a dual-processor Linux/PC workstation. The best algorithm evolved produced a cloud mask with an excellent match to the hand-drawn training data. Table I presents detection and false alarm rates and the chromosome fitness score (GENIE's internal measure of fitness, a floating-point number scaled from 0 to 1000) for the training case and several test cases, which we now describe. The training scene comprises part (a contiguous segment) of SCARB flight 95-163 track 7 with training data (i.e., pixel-scale cloud classification) provided by manual photo-interpretation. Test 1 comprises all of track 7 , again with manually marked-up training data. Test 2 comprises all of track 7 using the MODIS algorithm for training data. Test
TABLE I

EVOLVED CLOUD MASK PERFORMANCE

\begin{tabular}{llll} 
& \multicolumn{3}{c}{ EVOLVED CLOUD MASK PERFORMANCE } \\
\hline & $\begin{array}{l}\text { Detection } \\
\text { Rate [\%] }\end{array}$ & $\begin{array}{l}\text { False Alarm } \\
\text { Rate [\%] }\end{array}$ & Fitness \\
\hline Training & 99.8 & 0.38 & 997 \\
Test 1 & 99.5 & 0.0 & 997 \\
Test 2 & 93.2 & 14 & 895 \\
Test 3 & 98.3 & 8.1 & 980 \\
\hline
\end{tabular}

3 comprises all of flight 95-163 track 1 using a binary cloud mask produced by the MODIS algorithm for training data. As an extreme test of robustness, we also tested the algorithm on a scene from the TARFOX campaign, and the result was qualitatively very good, which we find encouraging for the future use of this machine learning technique.

The evolved algorithm works as follows: (A) a linear combination of MAS bands $1(0.546 \mu \mathrm{m}$ visible green/yellow) and $48(13.24 \mu \mathrm{m}$ thermal IR), spectrally equivalent to MODIS bands 4 and 33 (respectively), and close to MTI bands "B" $(0.55 \mu \mathrm{m}$ visible green/yellow $)$ and "N", are linearly combined to form the first intermediate feature plane. On inspection, this does a qualitatively good job of extracting the interiors of optically-thick clouds. (B) MAS band 2 $(0.653 \mu \mathrm{m}$ visible red), equivalent to MODIS band 1 and MTI band "C", undergoes an amplitude band-pass filter equivalent to a brightness test, which on inspection extracts the edges of bright clouds. (C) Finally, MAS band 7 $(0.865 \mu \mathrm{m}$ near IR), equivalent to MODIS band 2 and MTI band "E", undergoes local gradient spatial processing, equivalent to the difference of the standard morphological filtering operations of grayscale dilate and erode. This acts to extract the edges of clouds. A linear combination of these intermediate feature planes is then formed, with a vector of coefficients $(3.90,0.57,1.89)$ determined by the Fisher discriminant using the training data. The float-valued grayscale answer plane is then thresholded at a value 244.0, to produce a binary cloud mask, part of which is shown (for case Test 1, outside the training region) in Fig.1, right panel.

The noticeably higher false alarm rate in Test 2 deserves further comment. At its most pessimistic setting, the MODIS cloud mask tends to over-estimate the occurrence of clouds, relative to the human-specified simple-threshold algorithm. This is particularly noticeable at the edges of the MAS scenes (where geometric distortion of the image is quite visible, due to the large field-of-view of the MAS). The somewhat higher false alarm rate in this test case is due to the GENIE-evolved algorithm producing a result which does not show this effect, and which is closer to the simple-threshold method.

\section{DISCUSSION AND CONCLUSIONS}

The algorithm evolved by GENIE for this particular cloud mask application uses a thermal infrared band, a near IR 
band, and visible green/yellow and red bands. This is very similar to the method used with the human-specified simple-thresholds, though the band choices are not identical (GENIE also chooses to use MAS band 1 in intermediate feature plane A).

Fig. 1 shows a portion of the cloud image from the SCAR-B flight 95-163 track 7 data set. The left panel shows the clouds in the image using a near infrared band (similar to MTI band "E") from the MAS data (band 7). The right panel shows the cloud mask determined by GENIE. The MODIS and human-specified simple-threshold cloud masks are very similar to the GENIE result, and are not shown. Importantly, after training, the algorithm evolved by GENIE is fully automated relative to the simple-threshold technique, and executes in approximately 10 seconds on a complete MAS flight track, compared to a few minutes for the MODIS algorithm, using the same computer, though we should also take into account GENIE's initial training time (a few minutes to mark up the scene and 11 minutes of runtime, in this case).

We plan to continue the quantitative comparison of GENIE and the MODIS and MTI cloud masks in future work. Also, we plan to use both GENIE and the MTI cloud mask on a regular basis in detection products for the MTI spacecraft

\section{ACKNOWLEDGMENTS}

We wish to thank the MODIS cloud-mask team headed by Steve Ackerman at the University of Wisconsin, Madison for providing us with their cloud mask code. This work was supported by the U.S. Department of Defense, and by the Department of Energy under Contract W-7405-ENG-36.

\section{REFERENCES}

[1] P. G. Weber, B. C. Brock, A. J. Garrett, B. W. Smith, C. C. Borel, W. B. Clodius, S. C. Bender, R. R. Kay, and M. L. Decker, "Multispectral Thermal Imager mission overview," Proceedings of SPIE, vol. 3753, pp.394-402, 1999.

[2] K. Lewis Hirsch, S. P. Brumby, N. R. Harvey, and A. B. Davis, "The MTI Dense-Cloud Mask Algorithm Compared to a Cloud Mask Evolved by a Genetic Algorithm and to the MODIS Cloud Mask", Proc. SPIE, vol. 4132, pp. 83-90, 2000.

[3] M. D. King, W. P. Menze, P. S. Grant, J. S. Myers, G. T. arnod, S. E. Patnick, L. E. Gumey, S. C. Tsay, C. C. Moeler, M. Fitzgerald, K. S. Brown, and F. G. Osterwisch, "Airborne scanning spectrometer for remote sensing of cloud, aerosol, water vapor and surface properties," J. Atmos. Oceanic Technol., vol. 13, pp.777-794, 1996.

[4] W. B. Clodius, P. G. Weber, C. C. Borel, and B. W. Smith, "Multispectral band selection for satellite-based systems," Proc. SPIE, vol. 337, pp.11-21, 1998.

[5] S. A. Ackerman, K .I. Strabala, W. P. Menzel, R. A. Frey, C. C. Moeller, and L. E. Gumley, "Discriminating clear sky from clouds with MODIS,” J. Geophysical. Research, vol. 103, pp.32141-32158, 1998.

[6] S. P. Brumby, J. Theiler, S. J. Perkins, N. R. Harvey, J. J. Szymanski, J. J. Bloch, and M. Mitchell, "Investigation of feature extraction by a genetic algorithm," Proc. SPIE, vol. 3812, pp.24-31, 1999.

[7] GENIE is described at length on the GENIE Project web site: http://www.daps.lanl.gov/genie

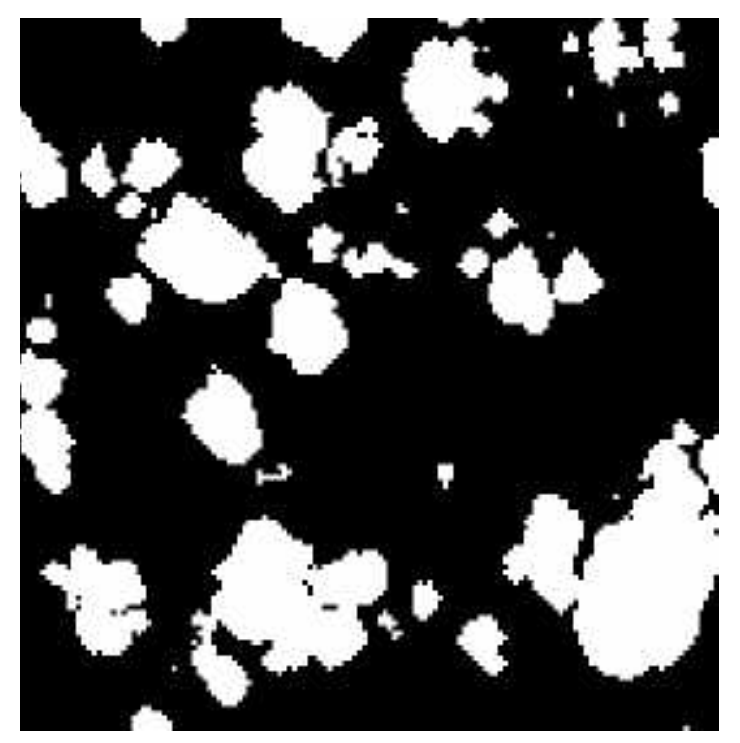

Fig. 1. A part of MAS band 7 of the training scene (left) is compared with the GENIE cloud mask algorithm result (right). 